\title{
Magnetic response from constant backgrounds to Coulomb sources
}

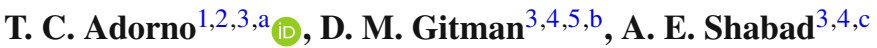 \\ ${ }^{1}$ Department of Physics, College of Physical Sciences and Technology, Hebei University, Wusidong Road 180, Baoding 071002, China \\ ${ }^{2}$ Key Laboratory of High-precision Computation and Application of Quantum Field Theory of Hebei Province, Hebei University, Baoding, China \\ ${ }^{3}$ Department of Physics, Tomsk State University, Lenin Prospekt 36, 634050 Tomsk, Russia \\ ${ }^{4}$ P. N. Lebedev Physical Institute, 53 Leninskiy prospekt, 119991 Moscow, Russia \\ ${ }^{5}$ Instituto de Física, Universidade de São Paulo, Caixa Postal 66318, São Paulo, SP CEP 05508-090, Brazil
}

Received: 30 September 2019 / Accepted: 17 March 2020 / Published online: 7 April 2020

(c) The Author(s) 2020

\begin{abstract}
Within general nonlinear electrodynamics given by a local action, very special magnetic field configuration carrying no magnetic charge is found as a linear response of the vacuum, filled with constant electric and magnetic fields, to an embedded static electric charge. When the electric charge is point-like and external fields are parallel, the response found may be interpreted as a field of two point-like magnetic charges of opposite polarities in one point. Coefficients characterizing the magnetic response and induced current are specialized to quantum electrodynamics (QED), where the nonlinearity is taken as that determined by the local Heisenberg-Euler effective action. It is demonstrated how the same response is reproduced in the (nonisotropic) region remote from the charge by considering the polarization operator of QED.
\end{abstract}

\section{Introduction}

It is well understood that the vacuum filled with strong background field is, in quantum electrodynamics (QED), equivalent to a linear or nonlinear medium [1-3]. Its properties are described by polarization 4-tensors of $n$-th rank defined as $\mathrm{n}$-th variational derivatives over fields of the effective action - their generating functional [4].

The second-rank polarization tensor $\Pi_{\mu \nu}(x, y)$ contains in itself linear polarization properties of the equivalent medium, usually referred to as dielectric permeability and magnetic permittivity. It is responsible for the screening of charges and currents and transformations of their shapes due

\footnotetext{
a e-mails: adorno@hbu.edu.cn; tg.adorno@gmail.com (corresponding author)

be-mail: gitman@if.usp.br

c e-mail: shabad@lpi.ru
}

to the strong background, and for small-amplitude electromagnetic wave propagation in the background, especially for polarization of the eigen-modes and (different) modifications of the mass shell in each mode, governing the birefringence that makes an important goal for observation [5-8]. The recent evidence for it is obtained from the neutron star RX J1856.5-3764 [9]).

The third-rank polarization tensor $\Pi_{\mu \nu \rho}(x, y, z)$, takes into account the quadratic response of the background. When taken on the photon mass shell, it is responsible for the photon splitting and merging in an external field [10-14]. Beyond the mass shell, it also describes the response of the medium to small perturbations with the quadratic accuracy relative to these perturbations. Analogously, the fourth-rank polarization tensor includes the cubic response, photon-by-photon scattering (the first experimental detection of this fundamental process, which is the source of nonlinearity of QED, was recently reported in Ref. [15]), photon splitting into three [14], and so on.

Usually, polarization tensors of any rank are meant to be calculated as Feynman diagrams in the Furry picture with solutions of the Dirac equation in external-field background taken for electron propagators. An essential simplification of calculations is achieved if one confines oneself to the local (infrared) approximation of the effective action, wherein the dependence of the latter on the space-time derivatives of the fields is disregarded. This approximation is good as long as the fields slowly varying in time and space are dealt with. Quite often, the Heisenberg-Euler expression calculated in one-loop [16] approximation in QED is used for the local effective action (also two-loop [17-22] and even three-loop [23] results are being considered). Within this approximation the field equations are differential (not integral) ones, and they do not include higher derivatives, while (the Fourier 
transform of) the polarization tensor of $n$-th rank behaves as the $n$-th power of the momentum; in the language of optics this corresponds to disregard of the spatial and frequency dispersion. ${ }^{1}$ The next step towards a better coverage of fastervarying fields is made by Gusynin and Shovkovy [26,27], who obtained an action functional that includes two derivatives of the field strength.

We thoroughly traced the derivation of the Maxwell equations within the local approximation in Refs. [28-32] in application to processes requiring the use of polarization tensors up to fourth rank. Interaction between long electromagnetic waves was considered taking into account, effectively, the polarization tensors up to 6-th rank in Refs. [33,34]. In Refs. $[30,35]$ we showed that the quadratic response of the vacuum with the background of a constant magnetic field to an applied electric field of a point-like or extended centralsymmetric charge, governed by the 3-rd rank polarization tensor is purely magnetic, i.e. we face here the magnetoelectric effect, where magnetic field is created by static electric charge. Moreover, the magnetic response far from the charge is the field of a magnetic dipole with its dipole moment quadratically dependent upon the electric charge. The photon splitting on the basis of the same diagram was studied in Ref. [14]. In Ref. [36] and now we take an arbitrary combination of constant electric $\overline{\mathbf{E}}$ and magnetic $\overline{\mathbf{B}}$ fields as a background (see [37,38] beyond the local approximation), and we consider linear response to an applied electric charge following the information contained in the 2-nd rank polarization tensor. This response may be both electric and magnetic. The electric response was studied in Ref. [36], resulting in description of the induced charge density and modification of the Coulomb field far from the charge. ${ }^{2}$

In the present paper we study the linear magnetic response of the constant background to an applied Coulomb source, complementary to our previous study [36], where only the linear electric response was found. Linearly induced currents and vector potentials are discussed in detail. Contrary to Ref. [39] and to our forthcoming work, only magnetic response with vanishing total magnetic charge is considered here. Correspondingly, if the Coulomb source is point-like, the finally found magnetic field looks like a combination of two opposite point-like magnetic charges coexisting in one point.

Applying the general results derived for any nonlinear theory to the special case where the nonlinearity is provided by QED at one-loop, we refer to relevant coefficients characterizing the results in terms of the Heisenberg-Euler effective

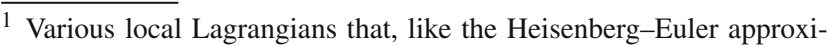
mation, do not contain space- and time-derivatives of fields, but are not associated with QED, are widely used together with the Einstein's gravity, especially when studying magnetized black holes. See e.g. [24,25] and pertinent references therein.

2 A brief review of our previous works may be found in Refs. [31,32].
}

action [16], whose proper-time representation and asymptotes in various parameter regimes are well known. Handier formulae are written under the simplifying assumption that the magnetic part of the background field is much larger that its electric part - the assumption of "magnetic dominance".

The magnetic response obtained is reliable only as long as the responded field is steady, since our approach is based on considering the local action functional. We demonstrate, in this connection, that starting from the "genuine" polarization tensor calculated in Refs. [37,38] as a loop of Dirac electron and positron propagators in external field and thereby free of the said limitation of applicability, we are able to reproduce the same magnetic response as a limit valid far from the point charge. The measure of remoteness, necessary for achieving this limit depends upon the direction. (This demonstration is performed for magnetic dominance case.)

The paper is organized as follows. In Sect. 2, after presenting the necessary Maxwell equations linearized near the background field and indicating the structure of the applied electric field, we obtain expressions for the current density induced in the "medium" inside and outside of the applied extended charge. In Sects. 2.1 and 2.2 we find the magnetic fields produced by this current and the vector potential corresponding to the magnetic response produced by a pointlike Coulomb source. All the results reported above are written in terms of the derivatives of the local effective Lagrangian over the field invariants taken at the background. Hence these may be used with every model Lagrangian, irrespective of its origin and of its connection to QED. On the contrary, in Sect. 3, we specialize the results to the one-loop EulerHeisenberg Lagrangian of QED. The proceeding beyond the local approximation is dealt with in Sect. 3.1. Section 4 is devoted to concluding remarks.

\section{Linearly induced currents and magnetic responses in constant backgrounds}

Let there be a background electromagnetic field, with its field tensor equal to $\bar{F}_{\nu \mu}(x)$, produced by the background current $\mathcal{J}_{\mu}$ via the (second set of) Maxwell equations ${ }^{3}$

$$
\begin{gathered}
\partial^{\nu} \bar{F}_{\nu \mu}(x)-\partial^{v}\left[\left.\frac{\delta \mathfrak{L}(\mathfrak{F}, \mathfrak{G})}{\delta \mathfrak{F}(x)}\right|_{F=\bar{F}} \bar{F}_{\nu \mu}(x)\right. \\
\left.+\left.\frac{\delta \mathfrak{L}(\mathfrak{F}, \mathfrak{G})}{\delta \mathfrak{G}(x)}\right|_{F=\bar{F}} \widetilde{\bar{F}}_{\nu \mu}(x)\right]=\mathcal{J}_{\mu}(x),
\end{gathered}
$$

\footnotetext{
${ }^{3}$ Greek indices span the 4-dimensional Minkowski space-time, e.g., $\mu=(0, i), i=1,2,3, \eta_{\mu \nu}=\operatorname{diag}(+1,-1,-1,-1)$, and boldface letters denote three-dimensional Euclidean vectors (e.g., $\mathbf{A}(x)=$ $\left(A^{i}(x), i=1,2,3\right)$. The four-rank and three-rank Levi-Civita tensors are normalized as $\varepsilon^{0123}=+1$ and $\varepsilon_{123}=1$, respectively.
} 
within a nonlinear local electrodynamics with the Lagrangian

$L(x)=-\mathfrak{F}(x)+\mathfrak{L}(x)$,

where its nonlinear part $\mathfrak{L}(x)$ is taken as a function $\mathfrak{L}(x)=$ $\mathfrak{L}(\mathfrak{F}, \mathfrak{G})$ of two field invariants $\mathfrak{F}=(1 / 4) F^{\mu v} F_{\mu \nu}=$ $(1 / 2)\left(\mathbf{B}^{2}-\mathbf{E}^{2}\right), \mathfrak{G}=(1 / 4) \tilde{F}^{\mu \nu} F_{\mu \nu}=-(\mathbf{E} \cdot \mathbf{B}), \tilde{F}_{\mu \nu}(x)$ $=(1 / 2) \varepsilon_{\mu \nu \alpha \beta} F^{\alpha \beta}(x)$, and may be thought of, for instance, as the effective Lagrangian of Quantum Electrodynamics in the local (infrared) approximation, i. e. the one where the dependence on the space- and time-derivatives of the fields is neglected. ${ }^{4}$ Moreover, we shall be considering the constant background $\bar{F}_{v \mu}(x)=\bar{F}_{v \mu}=$ const. here. This field does not require any current to be supported: it is seen that Eq. (1) is satisfied by $\mathcal{J}_{\mu}(x)=0$ in this case.

Let the constant background be disturbed by a small current $j_{\mu}(x)$. It causes the deviation $f_{v \mu}(x)=F_{v \mu}(x)-\bar{F}_{v \mu}$ of the field from the background. Expanding the Maxwell equations (1) in powers of $f_{v \mu}(x)$, we obtain in the first order the linear equation (see Refs. [28-32], [35,36,39] for equations for higher orders, which are nonlinear as containing the second and higher powers of $f_{v \mu}(x)$ ):

$$
\begin{aligned}
\partial^{v} f_{\nu \mu}(x)= & j_{\mu}^{\operatorname{lin}}(x)+j_{\mu}(x), \\
j_{\mu}^{\operatorname{lin}(x)=} & \partial^{\tau}\left[\mathfrak{L}_{\mathfrak{F}} f_{\tau \mu}(x)+\frac{1}{2}\left(\mathfrak{L}_{\mathfrak{F} \mathfrak{F}} \bar{F}_{\alpha \beta}\right.\right. \\
& \left.\left.+\mathfrak{L}_{\mathfrak{F} \mathfrak{G}} \widetilde{\bar{F}}_{\alpha \beta}\right) \bar{F}_{\tau \mu} f^{\alpha \beta}(x)\right] \\
& +\partial^{\tau}\left[\mathfrak{L}_{\mathfrak{G} \mathfrak{f}} \tilde{f}_{\tau \mu}(x)+\frac{1}{2}\left(\mathfrak{L}_{\mathfrak{F} \mathfrak{G}} \bar{F}_{\alpha \beta}\right.\right. \\
& \left.\left.+\mathfrak{L}_{\mathfrak{G} \mathfrak{G}} \widetilde{\bar{F}}_{\alpha \beta}\right) \tilde{\bar{F}}_{\tau \mu} f^{\alpha \beta}(x)\right],
\end{aligned}
$$

where the subscripts by $\mathfrak{L}$ designate derivatives with respect to the indicated field invariants taken at their background value, for instance $\left.\frac{\partial^{2} \mathfrak{L}}{\partial \mathfrak{F} \partial \mathfrak{G}}\right|_{F=\bar{F}}=\mathfrak{L}_{\mathfrak{F} \mathfrak{G}}$. We have introduced here the notation for the linearly induced current $j_{\mu}^{\text {lin }}(x)$ (nonlinearly induced currents were dealt with in Refs. $[28-32,35,36,39])$. To avoid possible misunderstanding, we stress that nonlinearly induced currents are responsible for self-induction of the deviation fields $f_{v \mu}(x)$, whereas the nonlinearity of the theory, given by the Lagrangian (2), shows itself in the present framework as the interaction between the electromagnetic field $f_{v \mu}(x)$ and the electromagnetic background $\bar{F}_{\alpha \beta}$.

In what follows we solve Eq. (3) perturbatively with respect to the above coefficients, whose connection with QED will be exploited in Sect. 3. To this end we represent

\footnotetext{
4 The special case with the Euler-Heisenberg Lagrangian, accepted as the one-loop approximation of such local effective action, will be treated in Sect. 3.
}

the electromagnetic field-strength tensor as

$f_{v \mu}(x)=f_{v \mu}^{(0)}(x)+f_{v \mu}^{(1)}(x)+\cdots$,

where $f_{v \mu}^{(0)}(x)$ is a solution of the classical field equation

$\partial^{v} f_{v \mu}^{(0)}(x)=j_{\mu}(x)$,

For the perturbation of the background we take the current $j_{\mu}(x)$ corresponding to a static charge $q$ homogeneously distributed over a ball with the radius $R$. Hence the null approximation $f_{v \mu}^{(0)}(x)$ includes $^{5}$

$$
\begin{aligned}
& \mathbf{E}^{(0)}(\mathbf{r})=\mathbf{E}_{\text {in }}^{(0)}(\mathbf{r}) \theta(R-r)+\mathbf{E}_{\text {out }}^{(0)}(\mathbf{r}) \theta(r-R), \\
& \mathbf{E}_{\text {in }}^{(0)}(\mathbf{r})=\frac{q \mathbf{r}}{4 \pi R^{3}}, \\
& \mathbf{E}_{\text {out }}^{(0)}(\mathbf{r})=\frac{q \mathbf{r}}{4 \pi r^{3}}, \quad \mathbf{B}^{(0)}(\mathbf{r})=0 .
\end{aligned}
$$

Throughout the text, the indexes "in" and "out" classify electromagnetic quantities at points inside $(r<R)$ and outside $(r \geq R)$ of the spherical charge distribution, respectively. In our previous work [36], we studied the electric response $E^{(1) k}(\mathbf{r}) \neq 0$,

$B^{(1) k}(\mathbf{r})=-(1 / 2) \varepsilon_{i j k} f^{(1) j k}(\mathbf{r})=0$ giving a correction to the Coulomb law (5); now we shall consider the purely magnetic solution $B^{(1) k}(\mathbf{r})=-(1 / 2) \varepsilon_{i j k} f^{(1) j k}(\mathbf{r}) \neq 0$, $E^{(1) k}(\mathbf{r})=0$. In Ref. [36] it was found that the first-order linear magnetic response $\mathbf{B}^{(1)}(\mathbf{r})$ to the purely electric perturbation $j_{\mu}(x)$ is the solution to the differential equation

$\nabla \times\left[\mathbf{B}^{(1)}(\mathbf{r})-\mathfrak{H}^{(0)}(\mathbf{r})\right]=0$,

where $\mathfrak{H}^{(0)}(\mathbf{r})$ is the expression within the brackets in (3), taken in the zeroth order, i.e. on solutions (5):

$\mathfrak{H}^{(0)}(\mathbf{r})=\mathfrak{H}_{\text {in }}^{(0)}(\mathbf{r}) \theta(R-r)+\mathfrak{H}_{\text {out }}^{(0)}(\mathbf{r}) \theta(r-R)$,

where

$$
\begin{aligned}
\mathfrak{H}_{\text {in }}^{(0)}(\mathbf{r}) & =\frac{q}{4 \pi R^{3}}\left\{-\mathfrak{L}_{\mathfrak{G}} \mathbf{r}-\left[\mathfrak{L}_{\mathfrak{F} \mathfrak{F}}(\overline{\mathbf{E}} \cdot \mathbf{r})+\mathfrak{L}_{\mathfrak{F} \mathfrak{G}}(\overline{\mathbf{B}} \cdot \mathbf{r})\right] \overline{\mathbf{B}}\right. \\
& \left.+\left[\mathfrak{L}_{\mathfrak{F} \mathfrak{G}}(\overline{\mathbf{E}} \cdot \mathbf{r})+\mathfrak{L}_{\mathfrak{G} \mathfrak{G}}(\overline{\mathbf{B}} \cdot \mathbf{r})\right] \overline{\mathbf{E}}\right\}, \quad r<R, \quad(8) \\
\mathfrak{H}_{\text {out }}^{(0)}(\mathbf{r}) & =\frac{q}{4 \pi r^{3}}\left\{-\mathfrak{L}_{\mathfrak{G}} \mathbf{r}-\left[\mathfrak{L}_{\mathfrak{F} \mathfrak{F}}(\overline{\mathbf{E}} \cdot \mathbf{r})+\mathfrak{L}_{\mathfrak{F} \mathfrak{G}}(\overline{\mathbf{B}} \cdot \mathbf{r})\right] \overline{\mathbf{B}}\right. \\
& \left.+\left[\mathfrak{L}_{\mathfrak{F} \mathfrak{G}}(\overline{\mathbf{E}} \cdot \mathbf{r})+\mathfrak{L}_{\mathfrak{G} \mathfrak{G}}(\overline{\mathbf{B}} \cdot \mathbf{r})\right] \overline{\mathbf{E}}\right\}, \quad r \geq R . \quad(9)
\end{aligned}
$$

Here and in what follows, the space- and time-independent electric and magnetic components of the background field are barred: $\bar{E}^{i}=\bar{F}_{0 i}, \bar{B}^{i}=-(1 / 2) \varepsilon_{i j k} \bar{F}^{j k}$.

\footnotetext{
${ }_{5}$ Hereafter $\theta(z)$ denotes the Heaviside step function defined as $\theta(z)=$ 1 if $z \geq 0$ and zero otherwise.
} 
Consider the linearly induced current density (3) to the same first-order approximation. According to Eq. (6), it is

$$
\begin{aligned}
& \mathbf{j}^{(1)}(\mathbf{r})=\nabla \times \mathfrak{H}^{(0)}(\mathbf{r})=\theta(R-r)\left[\nabla \times \mathfrak{H}_{\text {in }}^{(0)}(\mathbf{r})\right] \\
& \quad+\theta(r-R)\left[\boldsymbol{\nabla} \times \mathfrak{H}_{\text {out }}^{(0)}(\mathbf{r})\right] .
\end{aligned}
$$

Note that the quantity (7) is continuous at the border of the charge $r=R$, because $\mathbf{E}^{(0)}(\mathbf{r})(5)$ is. For this reason the differentiation of the step functions has not contributed to the sum (10). Hence, there does not appear any current at the surface of the charge. ${ }^{6}$ As a result, we may define the inner $\mathbf{B}_{\text {in }}^{(1)}(\mathbf{r})$ and the outer $\mathbf{B}_{\text {out }}^{(1)}(\mathbf{r})$ magnetic responses as solutions to the equations

$\boldsymbol{\nabla} \times \mathbf{B}_{\text {in }}^{(1)}(\mathbf{r})=\mathbf{j}_{\text {in }}^{(1)}(\mathbf{r}), \quad \nabla \times \mathbf{B}_{\text {out }}^{(1)}(\mathbf{r})=\mathbf{j}_{\text {out }}^{(1)}(\mathbf{r})$,

where the inner and outer parts of the first-order linearly induced current densities are

$$
\begin{gathered}
\mathbf{j}_{\text {in }}^{(1)}(\mathbf{r})=\nabla \times \mathfrak{H}_{\text {in }}^{(0)}(\mathbf{r})=\frac{q}{4 \pi R^{3}}\left(\mathfrak{L}_{\mathfrak{F F}}\right. \\
+\mathfrak{L}_{\mathfrak{G} \mathfrak{G})}[\overline{\mathbf{B}} \times \overline{\mathbf{E}}], \quad r<R,
\end{gathered}
$$

and

$$
\begin{aligned}
\mathbf{j}_{\text {out }}^{(1)}(\mathbf{r}) & \\
= & \nabla \times \mathfrak{H}_{\text {out }}^{(0)}(\mathbf{r}) \\
= & \frac{q}{4 \pi r^{3}}\left\{\mathfrak{L}_{\mathfrak{F} \mathfrak{F}}\left([\overline{\mathbf{B}} \times \overline{\mathbf{E}}]+\frac{3}{r^{2}}(\overline{\mathbf{E}} \cdot \mathbf{r})[\mathbf{r} \times \overline{\mathbf{B}}]\right)\right. \\
& +\mathfrak{L}_{\mathfrak{G} \mathfrak{G}}\left([\overline{\mathbf{B}} \times \overline{\mathbf{E}}]-\frac{3}{r^{2}}(\overline{\mathbf{B}} \cdot \mathbf{r})[\mathbf{r} \times \overline{\mathbf{E}}]\right) \\
& \left.+\frac{3}{r^{2}} \mathfrak{L}_{\mathfrak{F} \mathfrak{G}}((\overline{\mathbf{B}} \cdot \mathbf{r})[\mathbf{r} \times \overline{\mathbf{B}}]-(\overline{\mathbf{E}} \cdot \mathbf{r})[\mathbf{r} \times \overline{\mathbf{E}}])\right\}, \quad r \geq R .
\end{aligned}
$$

respectively. The induced current (10) is discontinuous at the edge of the ball, the same as the charge density is.

For the special case of parallel external backgrounds $\overline{\mathbf{B}} \|$ $\overline{\mathbf{E}}$, the induced current density inside the charge disappears, $\mathbf{j}_{\text {in }}^{(1)}=0$, while the current $\mathbf{j}_{\text {out }}^{(1)}(\mathbf{r})$ circles the coordinate axis parallel to their common direction. Letting $\hat{\mathbf{z}}$ be the unit vector $(|\hat{\mathbf{z}}|=1)$ along the common direction of the external fields,

$\hat{\mathbf{z}}\|\overline{\mathbf{B}}\| \overline{\mathbf{E}}$, the current density $\mathbf{j}_{\text {out }}^{(1)}(\mathbf{r})$ acquires the form

$\mathbf{j}_{\text {out }}^{(1)}(\mathbf{r})=\frac{3 q}{4 \pi r^{5}} \tilde{g}(\hat{\mathbf{z}} \cdot \mathbf{r})[\mathbf{r} \times \hat{\mathbf{z}}]$,

\footnotetext{
${ }^{6}$ Suppose there is a function with the structure similar to (7) (representing a "field") $f(x)=\theta(x-a) F(x)+\theta(a-x) Q(x)$. It is continuous in the point $x=a$, once $F(a)=Q(a)$. Consider its derivative (representing a "current"): $f^{\prime}(x)=\theta(x-a) F^{\prime}(x)+\theta(a-$ $x) Q^{\prime}(x)+\delta(x-a) F(x)-\delta(a-x) Q(x)$. The part of $f^{\prime}(x)$ concentrated at $x=a$ ("the surface current") $\delta(x-a) F(x)-\delta(a-x) Q(x)=$ $\delta(x-a)(F(x)-Q(x))=\delta(x-a)(F(a)-Q(a))=0$ disappears due to the continuity of $f(x)$. The remaining part of the "current" $f^{\prime}(x)=\theta(x-a) F^{\prime}(x)+\theta(a-x) Q^{\prime}(x)$ is discontinuous provided $F^{\prime}(x) \neq Q^{\prime}(x)$ at $x=a$.
}

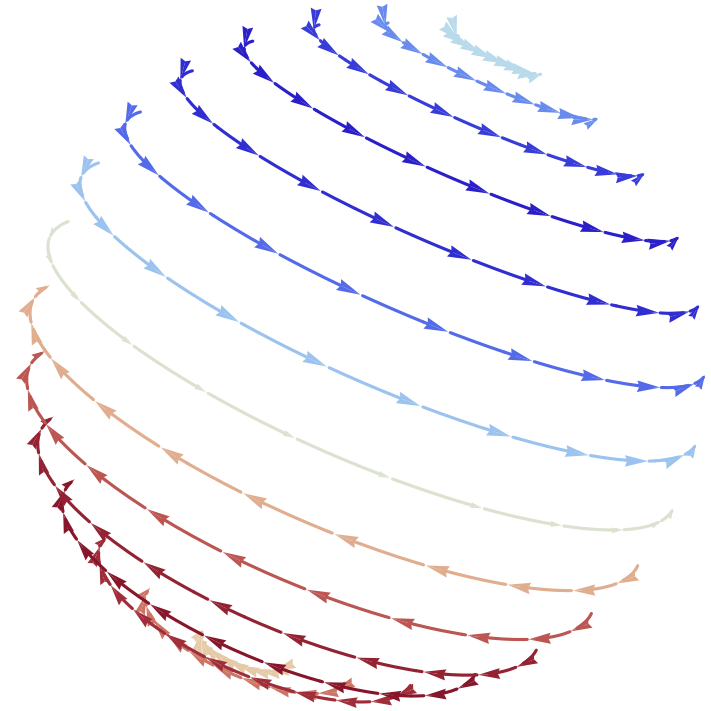

Fig. 1 Flux of the electric current (14) linearly induced by static electric charge placed into parallel electric and magnetic background fields (outside the charge). The current revolves about the axis drawn along the common direction of the background fields. The brighter the arrowhead lines, the larger the current density

where $\tilde{g}$ is a combination of derivatives of the effective Lagrangian and field invariants,

$$
\begin{aligned}
& \tilde{g}=\overline{\mathfrak{G}}\left(\mathfrak{L}_{\mathfrak{G} \mathfrak{G}}-\mathfrak{L}_{\mathfrak{F} \mathfrak{F}}\right)+2 \overline{\mathfrak{F}} \mathfrak{L}_{\mathfrak{F} \mathfrak{G}}, \\
& \overline{\mathfrak{G}}=-\overline{\mathbf{B}} \cdot \overline{\mathbf{E}}, \quad \overline{\mathfrak{F}}=\frac{1}{2}\left(\overline{\mathbf{B}}^{2}-\overline{\mathbf{E}}^{2}\right) .
\end{aligned}
$$

The current flux (14) flows in opposite directions in the upper and lower hemispheres; see Fig. 1. Hence, the total current through the part of a fixed meridional plane $\varphi=\varphi_{0}$, $0<\varphi_{0}<2 \pi$, enclosed between any two coordinate spheres $r_{1}<r<r_{2}$ is zero:

$\int \mathbf{j}_{\text {out }}^{(1)}(\mathbf{r}) d \mathbf{s}=\frac{3 q}{4 \pi} \tilde{g} \int_{r_{1}}^{r_{2}} \frac{d r}{r^{2}} \int_{0}^{\pi} d \theta \cos \theta \sin \theta=0$.

Here $\cos \theta=(\hat{\mathbf{z}} \cdot \mathbf{r}) / r$. Once any two mutually nonorthogonal constant fields may be reduced to parallelity by an appropriate Lorentz transformation to a special inertial frame, the current (13) differs from (14) by a contribution due to the motion of the charge in that frame.

\subsection{Magnetic response}

Besides the linearized Maxwell equations (6), the magnetic response $\mathbf{B}^{(1)}(\mathbf{r})$ should obey also the equation

$\nabla \cdot \mathbf{B}^{(1)}(\mathbf{r})=0$

that excludes an overall magnetic charge and makes the formulation of the theory in terms of potentials possible, in 
which case it corresponds to one of the Bianchi identities in electrodynamics. Equations (6) and (16) are satisfied by the magnetic response $\mathbf{B}^{(1)}(\mathbf{r})$

$B^{(1) i}(\mathbf{r})=\mathfrak{H}^{(0) i}(\mathbf{r})+\frac{1}{4 \pi} \partial_{i} \partial_{j} \int d \mathbf{y} \frac{\mathfrak{H}^{(0) j}(\mathbf{y})}{|\mathbf{r}-\mathbf{y}|}$.

The integrals on the rhs. of Eq. (17) can be evaluated following the method outlined in Appendix B in Ref. [36]. Their final expression can be obtained from Eqs. (B2) and (B6) of that reference after the substitutions $\mathfrak{L}_{\mathfrak{F}} \rightarrow-\mathfrak{L}_{\mathfrak{G}}$, $\mathfrak{L}_{\mathfrak{F} \mathfrak{F}} \bar{E}^{j} \rightarrow \mathfrak{L}_{\mathfrak{F} \mathfrak{F}} \bar{B}^{j}, \mathfrak{L}_{\mathfrak{F} \mathfrak{G}} \bar{B}^{j} \rightarrow-\mathfrak{L}_{\mathfrak{F} \mathfrak{G}} \bar{E}^{j}, \mathfrak{L}_{\mathfrak{F} \mathfrak{G}} \bar{E}^{j} \rightarrow$ $\mathfrak{L}_{\mathfrak{F} \mathfrak{G}} \bar{B}^{j}$ and $\mathfrak{L}_{\mathfrak{G} \mathfrak{G}} \bar{B}^{j} \rightarrow-\mathfrak{L}_{\mathfrak{G} \mathfrak{G}} \bar{E}^{j}$. As a result, the inner part $\mathbf{B}_{\text {in }}^{(1)}(\mathbf{r})$ takes the form

$$
\begin{aligned}
\mathbf{B}_{\text {in }}^{(1)}(\mathbf{r})= & \frac{q}{4 \pi}\left\{\left[\frac{3}{5 R^{3}} \mathfrak{L}_{\mathfrak{F} \mathfrak{G}}(\overline{\mathbf{E}} \cdot \mathbf{r})\right.\right. \\
& \left.+\frac{1}{5 R^{3}}\left(\mathfrak{L}_{\mathfrak{F} \mathfrak{F}}+4 \mathfrak{L}_{\mathfrak{G} \mathfrak{G}}\right)(\overline{\mathbf{B}} \cdot \mathbf{r})\right] \overline{\mathbf{E}} \\
& +\left[-\frac{3}{5 R^{3}} \mathfrak{L}_{\mathfrak{F} \mathfrak{G}}(\overline{\mathbf{B}} \cdot \mathbf{r})\right. \\
& \left.\left.-\frac{1}{5 R^{3}}\left(4 \mathfrak{L}_{\mathfrak{F} \mathfrak{F}}+\mathfrak{L}_{\mathfrak{G} \mathfrak{G}}\right)(\overline{\mathbf{E}} \cdot \mathbf{r})\right] \overline{\mathbf{B}}+\frac{\tilde{g}}{5 R^{3}} \mathbf{r}\right\},
\end{aligned}
$$

while the outer part $\mathbf{B}_{\text {out }}^{(1)}(\mathbf{r})$ can be conveniently written as

$\mathbf{B}_{\text {out }}^{(1)}(\mathbf{r})=\mathbf{B}_{\mathrm{pl}}^{(1)}(\mathbf{r})+\mathbf{B}_{\text {out }}^{(1)}(\mathbf{r} ; R)$.

Here $\mathbf{B}_{\mathrm{pl}}^{(1)}(\mathbf{r})$ denotes an $R$-free part

$$
\begin{aligned}
\mathbf{B}_{\mathrm{pl}}^{(1)}(\mathbf{r})= & \frac{q}{4 \pi}\left\{\frac{\tilde{g}}{2} \frac{\mathbf{r}}{r^{3}}-\frac{\left(\mathfrak{L}_{\mathfrak{G} \mathfrak{G}}+\mathfrak{L}_{\mathfrak{F} \mathfrak{F}}\right)}{2 r^{3}}[(\overline{\mathbf{E}} \cdot \mathbf{r}) \overline{\mathbf{B}}-(\overline{\mathbf{B}} \cdot \mathbf{r}) \overline{\mathbf{E}}]\right. \\
& +\frac{3}{2}\left[\left(\mathfrak{L}_{\mathfrak{G} \mathfrak{G}}-\mathfrak{L}_{\mathfrak{F} \mathfrak{F}}\right)(\overline{\mathbf{E}} \cdot \mathbf{r})(\overline{\mathbf{B}} \cdot \mathbf{r})\right. \\
& \left.\left.-\mathcal{L}_{\mathfrak{F} \mathfrak{G}}\left((\overline{\mathbf{B}} \cdot \mathbf{r})^{2}-(\overline{\mathbf{E}} \cdot \mathbf{r})^{2}\right)\right] \frac{\mathbf{r}}{r^{5}}\right\}
\end{aligned}
$$

while $\mathbf{B}_{\text {out }}^{(1)}(\mathbf{r} ; R)$, the $R$-dependent part, reads

$$
\begin{aligned}
\mathbf{B}_{\text {out }}^{(1)}(\mathbf{r} ; R)= & \frac{q}{8 \pi}\left(\frac{3 R^{2}}{5 r^{5}}\right)\left\{2 \mathfrak{L}_{\mathfrak{F} \mathfrak{G}}[(\overline{\mathbf{E}} \cdot \mathbf{r}) \overline{\mathbf{E}}-(\overline{\mathbf{B}} \cdot \mathbf{r}) \overline{\mathbf{B}}]\right. \\
& -\left(\mathfrak{L}_{\mathfrak{F F}}-\mathfrak{L}_{\mathfrak{G} \mathfrak{G}}\right)[(\overline{\mathbf{B}} \cdot \mathbf{r}) \overline{\mathbf{E}}+(\overline{\mathbf{E}} \cdot \mathbf{r}) \overline{\mathbf{B}}] \\
& +\left[-\tilde{g}+\frac{5}{r^{2}}\left(\mathfrak{L}_{\mathfrak{F F}}-\mathfrak{L}_{\mathfrak{G} \mathfrak{G}}\right)(\overline{\mathbf{E}} \cdot \mathbf{r})(\overline{\mathbf{B}} \cdot \mathbf{r})\right. \\
& \left.\left.+\frac{5}{r^{2}} \mathfrak{L}_{\mathfrak{F} \mathfrak{G}}\left((\overline{\mathbf{B}} \cdot \mathbf{r})^{2}-(\overline{\mathbf{E}} \cdot \mathbf{r})^{2}\right)\right] \mathbf{r}\right\}, \quad
\end{aligned}
$$

The division in $R$-dependent and $R$-free terms, expressed in Eq. (19), is aimed to emphasize that Eq. (21) corresponds to a pure homogeneous solution $\nabla \times \mathbf{B}_{\text {out }}^{(1)}(\mathbf{r} ; R)=0$. This is a consequence of the fact that the outer induced current density, given by Eq. (13), does not depend on $R$ or, in other words, there is no $R$-dependent source providing (21).
Its real role is to provide continuity of the whole magnetic response $\mathbf{B}^{(1)}(\mathbf{r})=\mathbf{B}_{\text {in }}^{(1)}(\mathbf{r}) \theta(R-r)+\mathbf{B}_{\text {out }}^{(1)}(\mathbf{r}) \theta(r-R)$ at the border of the Coulomb source. A similar feature has been reported by us in [36], wherein $R$-dependent terms in the electric response come automatically from the projection operator with the same interpretation. These $R$-dependent solutions are a consequence of the Coulomb source being an extended charge distribution rather than a pointlike one. In contrast, the $R$-independent part $\mathbf{B}_{\mathrm{pl}}^{(1)}(\mathbf{r})$ is the same as the first-order linear response of the pointlike Coulomb source, since it is the only survivor in the limit $r \gg R$ (or $R \rightarrow 0$ ), bearing in mind that for any non-special direction, $\mathbf{B}_{\mathrm{pl}}^{(1)}(\mathbf{r})$ decreases as $r^{-2}$, while $\mathbf{B}_{\text {out }}^{(1)}(\mathbf{r} ; R)$ decreases as $r^{-4}$. Therefore $\mathbf{B}_{\mathrm{pl}}^{(1)}(\mathbf{r})$ is identified as the first-order linear response to the pointlike Coulomb source. Moreover, according to Eq. $(13), \mathbf{B}_{\mathrm{pl}}^{(1)}(\mathbf{r})$ is provided by the outer induced current $\mathbf{j}_{\text {out }}^{(1)}(\mathbf{r})$

$\nabla \times \mathbf{B}_{\mathrm{pl}}^{(1)}(\mathbf{r})=\nabla \times \mathfrak{H}_{\text {out }}^{(0)}(\mathbf{r})=\mathbf{j}_{\text {out }}^{(1)}(\mathbf{r})$.

The first-order linear magnetic response calculated above does not carry any magnetic charge, as a consequence of the Bianchi identity (16). This is reflected in the triviality of the Gauss integral

$\oint_{S}\left(\mathbf{B}^{(1)}(\mathbf{r}) \cdot \hat{\mathbf{n}}\right) d S=0$,

for an arbitrary closed surface $S$ embracing the charge $q$. This integral vanishes for each magnetic response $\mathbf{B}_{\text {in }}^{(1)}(\mathbf{r})$, $\mathbf{B}_{\mathrm{pl}}^{(1)}(\mathbf{r})$ and $\mathbf{B}_{\text {out }}^{(1)}(\mathbf{r} ; R)$, independently. (We refer the reader to our arXiv preprint [40] for explicit demonstration of this statement and other details.) This implies that magnetic lines of force incoming to and outgoing from the charge $q$, compensate each other, so that the corresponding magnetic flux be zero.

To visualize the structure of the magnetic lines of force, let us consider the particular case of parallel background fields, $\overline{\mathbf{E}}=\bar{E} \hat{\mathbf{z}}, \quad \overline{\mathbf{B}}=\bar{B} \hat{\mathbf{z}}(|\hat{\mathbf{z}}|=1)$, whose response acquires a simpler form

$\mathbf{B}_{\text {in }}^{(1)}(\mathbf{r})=\frac{q \tilde{g}}{4 \pi R^{3}} \frac{1}{5}[\mathbf{r}-3(\hat{\mathbf{z}} \cdot \mathbf{r}) \hat{\mathbf{z}}]$,

and

$$
\begin{aligned}
\mathbf{B}_{\text {out }}^{(1)}(\mathbf{r})= & \frac{q \tilde{g}}{4 \pi r^{3}}\left\{\left[1-\frac{3 R^{2}}{5 r^{2}}-3\left(1-\frac{R^{2}}{r^{2}}\right)\left(\frac{\hat{\mathbf{z}} \cdot \mathbf{r}}{r}\right)^{2}\right] \frac{\mathbf{r}}{2}\right. \\
& \left.-\frac{3 R^{2}}{5 r^{2}}(\hat{\mathbf{z}} \cdot \mathbf{r}) \hat{\mathbf{z}}\right\} .
\end{aligned}
$$

Moreover, in the limit $r / R \rightarrow \infty$, we are left with a single magnetic response, exclusively radial, although spherically 
nonsymmetric, corresponding to that of a pointlike Coulomb source

$\mathbf{B}_{\mathrm{pl}}^{(1)}(\mathbf{r})=\lim _{r / R \rightarrow \infty} \mathbf{B}^{(1)}(\mathbf{r})=\frac{q}{4 \pi} \frac{\tilde{g}}{2}\left[1-3\left(\frac{\hat{\mathbf{z}} \cdot \mathbf{r}}{r}\right)^{2}\right] \frac{\mathbf{r}}{r^{3}}$.

The magnetic lines of force are straight lines, vanishing at the angles $\cos \theta=\zeta=\zeta_{0}=1 / \sqrt{3}$. As no net magnetic charge exists for producing a nontrivial magnetic flux, there are inward magnetic lines (pointing to $q$ ) and outward magnetic lines (lines leaving $q$ ), in the same proportion (see Fig. $2)$.

The magnetic field found (26) may be understood as two pointlike magnetic poles of equal, but opposite, polarities superposed in one point, so to say, a pointlike magnetic dipole.

\subsection{Vector potentials}

In this subsection, we extend the above consideration to the level of electromagnetic potentials, restricting ourselves to the case of electromagnetic responses generated by a pointlike charge distribution since, as discussed before, the role of the regularization ) is simply to avoid divergent integrals in the calculation of magnetic responses (17) if a pointlike source is considered from the beginning. In this case, the vector potential is sought in the form, ${ }^{7}$

$$
\begin{aligned}
& \mathbf{A}^{(1)}(\mathbf{r})=[\hat{\mathbf{z}} \times \mathbf{r}] \frac{\mathcal{A}(\zeta, \xi)}{r^{2}}+[\hat{\mathbf{v}} \times \mathbf{r}] \frac{\mathcal{C}(\zeta, \xi)}{r^{2}}+\frac{[\hat{\mathbf{z}} \times \hat{\mathbf{v}}]}{r} \mathcal{N}, \\
& \hat{\mathbf{z}}=\frac{\overline{\mathbf{B}}}{\bar{B}}, \hat{\mathbf{v}}=\frac{\overline{\mathbf{E}}}{\bar{E}}, \quad \zeta=\frac{\hat{\mathbf{z}} \cdot \mathbf{r}}{r}, \quad \xi=\frac{\hat{\boldsymbol{v}} \cdot \mathbf{r}}{r},
\end{aligned}
$$

where $\mathcal{N}$ is a constant and $\mathcal{A}(\zeta, \xi), \mathcal{C}(\zeta, \xi)$ are functions of the cosines of the angles between directions of $\overline{\mathbf{E}}, \overline{\mathbf{B}}$ and the radius vector $\mathbf{r}$. Although this form may be not the most general one, its use is sufficient for finding at least a certain class of the vector-potentials $\mathbf{A}^{(1)}(\mathbf{r})$, all the variety of other possible values for $\mathbf{A}^{(1)}$ (r) being gauge-equivalent to those found. The fulfillment of the program of finding the vector potentia in the form (27) is presented in detail in Ref. [40]. It includes solving a first-order partial differential equation for two functions $\mathcal{A}(\zeta, \xi)$ and $\mathcal{C}(\zeta, \xi)$. Boundary conditions for this equation span different choices of the gauge. There is a remarkable choice that results in an expression for $\mathbf{A}^{(1)}$ (r) (27), free of the singularity along directions of the two background fields specified by $\xi, \zeta= \pm 1$

\footnotetext{
${ }^{7}$ For the sake of convenience, we remove the subscript "pl" on the magnetic response generated by a pointlike Coulomb distribution, given by Eq. (20). Thus $\mathbf{B}_{\mathrm{pl}}^{(1)}(\mathbf{r}) \equiv \mathbf{B}^{(1)}(\mathbf{r})$ from now on.
}

$$
\begin{aligned}
\mathbf{A}^{(1)}(\mathbf{r})= & \frac{q}{16 \pi r^{2}}[\overline{\mathbf{B}} \times \mathbf{r}] \\
& \times\left[\left(\mathfrak{L}_{\mathfrak{G} \mathfrak{G}}-\mathfrak{L}_{\mathfrak{F} \mathfrak{F}}\right)\left(\frac{\overline{\mathbf{E}} \cdot \mathbf{r}}{r}\right)-2 \mathfrak{L}_{\mathfrak{F} \mathfrak{G}}\left(\frac{\overline{\mathbf{B}} \cdot \mathbf{r}}{r}\right)\right] \\
& +\frac{q}{16 \pi r^{2}}[\overline{\mathbf{E}} \times \mathbf{r}] \\
& \times\left[\left(\mathfrak{L}_{\mathfrak{G} \mathfrak{G}}-\mathfrak{L}_{\mathfrak{F} \mathfrak{F}}\right)\left(\frac{\overline{\mathbf{B}} \cdot \mathbf{r}}{r}\right)+2 \mathfrak{L}_{\mathfrak{F} \mathfrak{G}}\left(\frac{\overline{\mathbf{E}} \cdot \mathbf{r}}{r}\right)\right] \\
& +\frac{q}{8 \pi}\left(\mathfrak{L}_{\mathfrak{F} \mathfrak{F}}+\mathfrak{L}_{\mathfrak{G} \mathfrak{G})} \frac{[\overline{\mathbf{B}} \times \overline{\mathbf{E}}]}{r^{2}},\right.
\end{aligned}
$$

which does not obey the Coulomb gauge condition

$\nabla \cdot \mathbf{A}^{(1)}(\mathbf{r})=\frac{q\left(\mathfrak{L}_{\mathfrak{F F}}+\mathfrak{L}_{\mathfrak{G} \mathfrak{G}}\right)}{8 \pi} \frac{\mathbf{r} \cdot[\overline{\mathbf{E}} \times \overline{\mathbf{B}}]}{r^{3}}$,

on account of the non-parallelism between the background fields. For parallel backgrounds, Eq. (28) reduces to

$\mathbf{A}^{(1)}(\mathbf{r})=-\frac{q}{4 \pi} \frac{[\hat{\mathbf{z}} \times \mathbf{r}]}{r^{2}} \frac{\tilde{g}}{2} \zeta$,

This is subject to the Coulomb gauge condition $\nabla \cdot \mathbf{A}^{(1)}(\mathbf{r})=0$.

\section{Results in QED}

To visualize how the magnetic responses and related effects, valid for any local nonlinear theory, depend on the constant background, we apply the former results to a specific theory, whose nonlinearity is provided by the local approximation of the effective Lagrangian of QED found within one-fermionloop calculation by Heisenberg and Euler [16] (see e.g. [41])

$$
\begin{aligned}
\mathfrak{L} & =\frac{m_{\mathrm{e}}^{4}}{8 \pi^{2}} \int_{0}^{\infty} d t \frac{e^{-t}}{t^{3}}\{-(t a \cot t a)(t b \operatorname{coth} t b) \\
& \left.+1-\frac{1}{3}\left(a^{2}-b^{2}\right) t^{2}\right\},
\end{aligned}
$$

where the integration contour is meant to circumvent the poles on the real axis of $t$ supplied by $\cot t a$, above the real axis. Here $a=\left(e / m_{\mathrm{e}}^{2}\right) \sqrt{-\overline{\mathfrak{F}}+\sqrt{\overline{\mathfrak{F}}^{2}+\overline{\mathfrak{G}}^{2}}}$ and $b=$

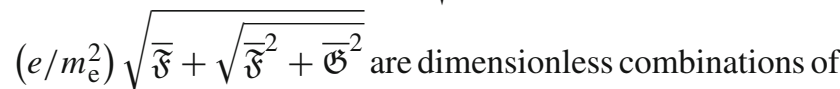
the field invariants, and have the meaning of the electric and magnetic field in the Lorentz frame where these are parallel, normalized to the characteristic field value $m_{\mathrm{e}}^{2} / e$. Here $m_{\mathrm{e}}^{2}$ and $e$ are the electron mass and charge (in absolute value), respectively. As it is well known, such a frame always exists when $\overline{\mathfrak{G}} \neq 0$.

We are primarily interested in strong magnetic-dominated backgrounds, in which the electric contribution is sufficiently small in comparison to the magnetic part 


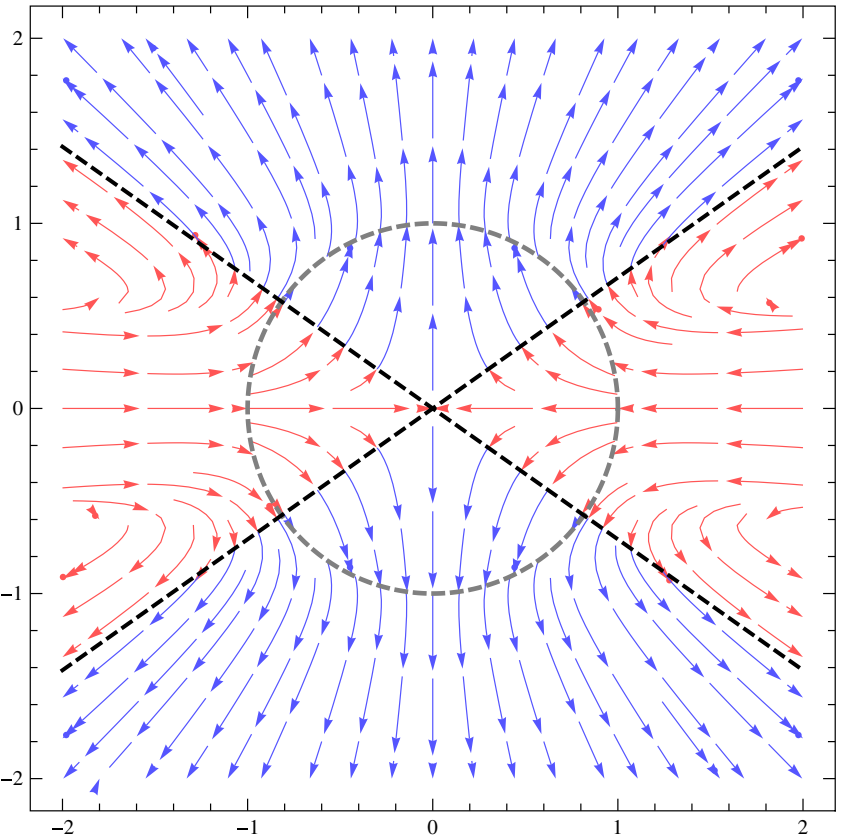

Fig. 2 Magnetic lines of force produced in a constant background by the static charge. The left pattern corresponds to the extended charge concentrated within the dashed circle, and the right pattern corresponds to the point charge [according to Eq. (26)]. Magnetic field $\mathbf{B}_{\text {in }}^{(1)}(\mathbf{x})$ inside the charge is drawn following Eq. (24), and its outer part $\mathbf{B}_{\text {out }}^{(1)}(\mathbf{x})$ following Eq. (25). In the limit $R \rightarrow 0$,

$\frac{a}{b} \ll 1$

irrespective of whether $a$ and $b$ are small or not as compared to the unity, implying the magnetic dominance $\overline{\mathbf{B}} \gg \overline{\mathbf{E}}$ in any reference frame. Such condition is enough to probe vacuum nonlinear effects ${ }^{8}$, and should be applied in final expressions after all coefficients composing the magnetic responses, in particular $\tilde{g}$ (15), have been calculated. The necessary second derivatives of the effective Lagrangian (31) with $a$ and $b$ arbitrary, i.e. not subjected to condition (32), are first obtained as Eqs. (11) in Ref. [43] [see also Eqs. (51)-(54) in Ref. [36] ]. The corresponding expressions are sufficient to form $\tilde{g}$, but the arising integral representations are overcomplicated. For this reason, we address ourselves to their magnetic-dominance limits, given by Eqs. (58) in Ref. [36]. ${ }^{9}$ These expressions are obtained by expanding the trigonometric functions in the integral representations following from (31) into ascending powers of $a / b$. In this way the leading

\footnotetext{
${ }^{8}$ Note that the magnetic responses (18), (19) and the nonlinearly induced currents (12), (13), vanish identically in the pure magnetic background $a / b=0$, since $\overline{\mathfrak{G}}=0$.

9 The remaining coefficient $\mathfrak{F}_{\mathfrak{F} \mathfrak{G}}^{(1)}$ resulting from the expansion $\mathfrak{F}_{\mathfrak{F} \mathfrak{G}}=(a / b) \mathfrak{F}_{\mathfrak{F} \mathfrak{G}}^{(1)}+O\left(a^{2} / b^{2}\right)$ formally coincides with $\left(\mathfrak{G} \mathfrak{L}_{\mathfrak{F} \mathfrak{G}}\right)^{(2)}$ in Ref. [36] except by a multiplying factor of $\kappa / 2$, namely $\mathfrak{F} \mathfrak{L}_{\mathfrak{F} \mathfrak{G}}^{(1)}=$ $(\kappa / 2)\left(\mathfrak{G} \mathfrak{L}_{\mathfrak{F} \mathfrak{G}}\right)^{(2)}$.
}

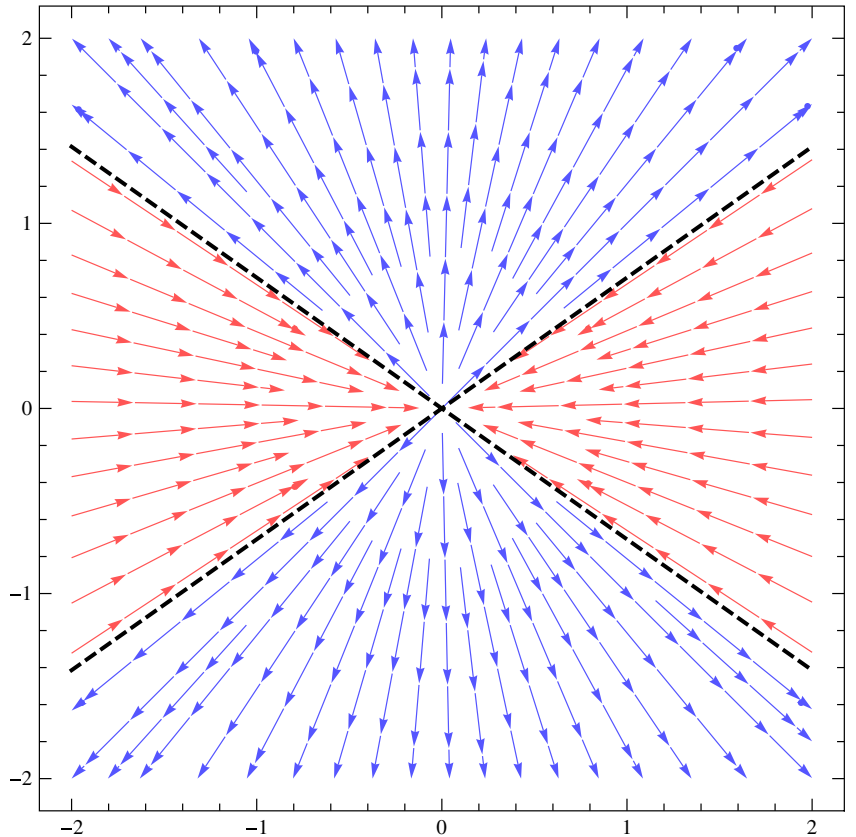

the red/blue arrows in the left pattern tend to straight lines, becoming "inward" and "outward" magnetic lines of force, as depicted in the right pattern. The inclined dashed lines indicate regions of zero magnetic field on the right, whereas, on the left, they only divide "inward" and "outward" magnetic lines of force in the limit $R \rightarrow 0$

term $\tilde{g}^{(1)}$ in the expansion $\tilde{g}=(a / b) \tilde{g}^{(1)}+O\left((a / b)^{3}\right)$, is obtained:

$$
\begin{aligned}
\tilde{g}^{(1)}= & \kappa\left(\frac{\alpha}{2 \pi}\right) \int_{0}^{\infty} d \tau \frac{e^{-\tau / b}}{\tau}\left[\frac{1}{\tau} \operatorname{coth} \tau\right. \\
& \left.-\left(1+\frac{2 \tau^{2}}{3}\right) \frac{1}{\sinh ^{2} \tau}\right], \quad \kappa=\operatorname{sgn}(\overline{\mathfrak{G}}) .
\end{aligned}
$$

In the magnetic dominance case, the same as for the purely magnetic background, it is possible to express all above integrals in terms of the Hurwitz Zeta function $\zeta(z, \sigma)$, DiGamma function $\psi(z)=\Gamma^{\prime}(z) / \Gamma(z)$ and related functions [42]. The Zeta-function representations for all the necessary derivatives of the Heisenberg-Euler effective Lagrangian can be found in Ref. [43]. The missing representation - corresponding to $\mathfrak{F} \mathfrak{L}_{\mathfrak{F} \mathfrak{G}}$ - can be written analogously. Using Zeta-function regularization techniques (see e. g., [44-46]), $\tilde{g}^{(1)}$ is expressed as follows

$$
\begin{aligned}
\tilde{g}^{(1)}= & \kappa\left(\frac{\alpha}{2 \pi}\right)\left\{\frac{1}{2 b^{2}}-\frac{1}{3}+\frac{2}{3} \psi\left(\frac{1}{2 b}\right)-8 \zeta^{\prime}\left(-1, \frac{1}{2 b}\right)\right. \\
& \left.+\frac{1}{b}\left[\log \frac{b}{\pi}+2 \log \Gamma\left(\frac{1}{2 b}\right)+\frac{1}{3} \psi^{(1)}\left(\frac{1}{2 b}\right)\right]\right\},
\end{aligned}
$$

where $\zeta^{\prime}(z, \sigma)$ is the derivative of the Hurwitz Zeta function with respect to $z, \psi^{(j)}(z)$ is the $j$-th derivative of the DiGamma function and $\gamma \approx 0.577$ is the Euler constant 
[42]. Using asymptotic representations of special functions appearing in Eq. (34), (see e.g., [44-47]) the above coefficient tends to the constant for growing magnetic field:

$$
\begin{aligned}
& \tilde{g}^{(1)} \sim-\kappa\left(\frac{\alpha}{2 \pi}\right)\left(\frac{1}{3}+\frac{2}{3} \gamma+8 \zeta^{\prime}(-1)\right) \\
& \approx \kappa\left(\frac{\alpha}{2 \pi}\right) 0.60523, \quad b \rightarrow \infty,
\end{aligned}
$$

Therefore, the leading-order contribution to $\tilde{g}=(a / b) \tilde{g}^{(1)}+$ $O\left((a / b)^{3}\right)$ vanishes in the limit of infinite magnetic field. The linearly growing with the magnetic field contribution from $\mathfrak{L}_{\mathfrak{G} \mathfrak{G}}$ - known to be responsible for growing electric response and growing screening of the Coulomb field of the nucleus in an atom - is cancelled in $\tilde{g}$ by the analogous contri-

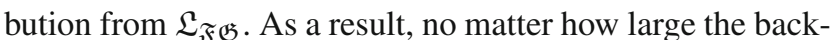
ground may be (as long as the condition (32) is preserved), the perturbation parameter for the linear magnetic response $\sim(\alpha / 2 \pi) \times(a / b)$ remains small or tends to zero. Therefore, the one-loop accuracy of $\alpha$ laid in this result is respected for any magnitude of the magnetic-dominant background.

\subsection{Magnetic response as a large-distance limit from} results beyond the infrared approximation

Since the infrared approximation restricts the consideration to slow-varying electromagnetic fields and at points sufficiently close to the charge distribution where the electric
$A_{i}(\mathbf{x})=\frac{q}{(2 \pi)^{3}} \int D_{i 0}(\mathbf{k}) \exp (-i \mathbf{k x}) d^{3} k$

where $D_{i 0}(\mathbf{k})$ is the static limit of the photon propagator in the background field, subject to the decomposition

$D_{i 0}(\mathbf{k})=\sum_{a=1,2,3} \frac{b_{i}^{(a)} b_{0}^{(a)}}{\left(b^{(a)}\right)^{2}} \frac{1}{\mathbf{k}^{2}+\varkappa_{a}}$,

in terms of 4-eigenvectors $\mathrm{b}_{\mu}^{(a)}$ and eigenvalues $\varkappa_{a}$ of the mentioned polarization tensor. It was shown in Ref. [38] that only components numbered as "2" and " 3 " in this decomposition give rise to magnetic field of the charge $q$. In the regime of magnetic dominance under consideration one has for $(36)^{10}$

$$
\begin{aligned}
& \mathbf{A}(\mathbf{x})=\frac{-i q}{(2 \pi)^{3}} \int \mathbf{c}^{-} \frac{\Lambda_{3}(k) \exp (-i \mathbf{k x}) d^{3} k}{\left(\mathbf{k}^{2}+\varkappa_{2}\right)\left(\mathbf{k}^{2}+\varkappa_{3}\right)}, \\
& \mathbf{A}=\left(\mathbf{A}_{\perp}, A_{z}=0\right) .
\end{aligned}
$$

Here $\mathbf{c}^{-}=\left[\mathbf{k}_{\perp} \times \hat{\mathbf{z}}\right] /\left|\mathbf{k}_{\perp}\right|$, in which $\mathbf{k}_{\perp}$ denotes the projection of the photon 3-momentum $\mathbf{k}$ onto the plane orthogonal to the common orientation of the background fields, $\hat{\mathbf{z}}$. The Lorentz scalar $\Lambda_{3}$, as calculated to the first power in the fine-structure constant $\alpha$ (as the one-loop diagram), is given in the static limit $k_{0}=0$ in the rest frame of the charge (remind that we restrict ourselves to the case where the background fields are parallel in that frame) by the integral

$$
\begin{aligned}
\Lambda_{3}(k)= & -\frac{i \alpha}{4 \pi} \frac{\mathfrak{G}}{\mathfrak{F}}\left|\mathbf{k}_{\perp}\right| k_{\|} \int_{0}^{\infty} \frac{\tau d \tau}{\sinh ^{2} \tau} \int_{-1}^{1} d \eta(1-\eta)^{2} \sinh ^{2} \frac{\tau(1+\eta)}{2} \times \\
& \times \exp \left\{-\frac{k_{\perp}^{2}}{e B} \frac{\sinh \left[(1+\eta) \frac{\tau}{2}\right] \sinh \left[(1-\eta) \frac{\tau}{2}\right]}{\sinh \tau}-\frac{k_{\|}^{2}}{e B}\left(\frac{1-\eta^{2}}{4}\right) \tau-\frac{m_{\mathrm{e}}^{2}}{e B} \tau\right\} .
\end{aligned}
$$

perturbation is intrinsically fast-varying, it is essential to study conditions by which the present results are justified. To this aim we consider the exact approach developed in Refs. [37,38] specialized to the magnetic dominated regime (32). As we shall see below, the vector-potential (30) correspond to the asymptotic expression valid far from the charge for the linear magnetic response first calculated within the approach free of the limitation that the response should be slow-varying in space and time. Such approach is provided by the use of the photon polarization tensor taken as the oneloop diagram of the Furry picture formed by the electron and positron exact Dirac propagators in the constant external electric and magnetic field. The strength of that field is not restricted from above, and the polarization tensor is not derived from a local effective action. The needed polarization tensor beyond the photon mass shell, $k_{0}^{2}-\mathbf{k}^{2} \neq 0$, is known since $[37,38]$. The 3 -vector potential produced by a point-like charge $q$ is
Here $k_{\|}=(\mathbf{k} \hat{\mathbf{z}})$. Note that in accordance with the magnetic dominance adopted, the electric background field is present only as the factor $\mathfrak{G}$. Eqs. (37) and (38) supply us with the possibility of finding the linear magnetic response to a point charge free of the limitation that the field of this charge be slow-varying. Although both equations are sufficient for studying the singularity of the linear magnetic response near the charge, it is noteworthy that it corresponds going beyond the quadratic approximation studied in Sect. 2 , therefore beyond the scope of this work. Nevertheless, the present approach gives the necessary information regarding the distance from the point charge in comparison to elementary length scales.

To pass to large distances $\mathbf{x}_{\perp}, x_{\|}$, where the field of the charge is expected to be slow-varying, we have to consider the limit of small $\mathbf{k}$ in $\Lambda_{3}$. As seen from (38), the

\footnotetext{
$\overline{10}$ We have removed the unjustified factor $i$ in $c_{0}^{+}$from Eq. (31) of Ref. [38] to write the corrected Eq. (37).
} 
measures of smallness are different for $\mathbf{k}_{\perp}$ and $k_{\|}$. When $k_{\|}^{2} \ll m_{\mathrm{e}}^{2}$ and $k_{\perp}^{2} \ll e B$ (note, that the combination of hyperbolic sines in the exponential is a restricted function), we can neglect all momentum-depending terms in the exponent to get the asymptotic regime of large distances $x_{\|} \gg \lambda_{\mathrm{C}}$, $\left|\mathbf{x}_{\perp}\right| \gg(e B)^{-1 / 2}$, with $\lambda_{\mathrm{C}}$ being the electron Compton length, and $(e B)^{-\frac{1}{2}}$ its classical orbit radius in a magnetic field. Such are, therefore, the two formation lengths of the polarization tensor for two different directions in a magnetic field.

The knowledge of these restrictions is important when we need to enhance the rather small magnitude of the magnetic response (26), (30) as revealed in the discussion above. With the most favorable admitted values for the ratio $\frac{a}{b}$ taken to be of the order of $0.4 \div 0.3$, the coefficient $\widetilde{g}$ is only $(0.2 \div 0.15) \frac{\alpha}{2 \pi}$. To enhance the magnetic response we have to approach the point charge as close as the local approximation permits. Admitting that the magnetic part of the background field exceeds the characteristic value of $\frac{m^{2}}{e}$, that is that $b \gtrsim 1$ or $\lambda_{\mathrm{C}} \gtrsim(e \bar{B})^{-1 / 2}$, we are permitted in agreement with above to approach the charge along any direction down to the Compton length $\lambda_{\mathrm{C}}$. Then the maximum magnetic field of the response (26) will be of the order of ${ }^{11} B_{p l}^{(1)} \sim B_{c r} \widetilde{g}$ $\alpha Z \sim B_{c r} 10^{-1} \alpha^{2} Z$, where $Z$ is the charge number $Z=q / e$. Large charges are favorable.

After setting $k_{\|}^{2}=k_{\perp}^{2}=0$, the remaining $\eta$-integral in (38) can be readily calculated, leading to the following limit of $\Lambda_{3}$ :

$$
\left.\Lambda_{3}(k)\right|_{k_{\mu} \rightarrow 0}=-i \frac{\kappa \alpha}{2 \pi}\left(\frac{a}{b}\right)\left|\mathbf{k}_{\perp}\right| k_{\|} \int_{0}^{\infty} d \tau \frac{e^{-\tau / b}}{\tau} \times\left[\frac{\operatorname{coth} \tau}{\tau}-\left(1+\frac{2}{3} \tau^{2}\right) \frac{1}{\sinh ^{2} \tau}\right] .
$$

Bearing in mind the desired identity of the $\tau$-integral in (39) and (33), we can represent $\Lambda_{3}$ in the magnetic dominance regime as

$\Lambda_{3}(k)=-i\left|\mathbf{k}_{\perp}\right| k_{\|} \tilde{g}$

Using this in (37) we obtain for large distances from the point charge, $x_{\|} \gg \lambda_{\mathrm{C}}, x_{\perp} \gg(e B)^{-1 / 2}$,

$$
\left.\mathbf{A}\left(\mathbf{x}_{\perp}, x_{\|}\right)\right|_{|\mathbf{x}| \rightarrow \infty} \approx \frac{q \tilde{g}}{(2 \pi)^{3}} \int k_{\|}\left[\mathbf{k}_{\perp} \times \hat{\mathbf{z}}\right] \frac{\exp (-i \mathbf{k x}) d^{3} k}{\mathbf{k}^{4}}
$$

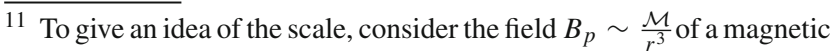
moment of 3 nuclear magnetons $\mathcal{M}=3 \frac{e}{2 m_{\mathrm{p}}}$, characteristic of the proton in the vacuum, at a distance $r=\lambda_{\mathrm{C}}$ from its centre, $B_{p} \sim \frac{3}{2} \frac{\mathrm{em} \mathrm{e}_{\mathrm{e}}^{3}}{m_{\mathrm{p}}} \approx$ $\frac{3}{2} \frac{e m_{\mathrm{e}}^{2}}{\left(m_{p} / m_{\mathrm{e}}\right)}=\frac{3 \pi \alpha B_{c r}}{10^{3}}$. This field is one order of magnitude larger than our magnetic response at $Z=1$, but it decays with distance following the cubic law as compared to quadratic law in (26).
}

In order to keep the same accuracy as the first term in expansion (4), adopted when solving Eq. (3), we disregarded contributions from $\varkappa_{2}, \varkappa_{3}$ to denominators as providing higher orders in $\alpha$.

Now we are in a position to compare the large-distance asymptotic behavior (41) with our Heisenberg-Euler-based result (30). It follows from the rotational covariance that Eq. (41) can be expressed in terms of an scalar integral $I(\mathbf{r} \hat{\mathbf{z}})$,

$I(\mathbf{r} \hat{\mathbf{z}})=\frac{1}{r^{2} \sin ^{2} \theta} \int k_{\|}\left(\mathbf{k}_{\perp} \mathbf{r}_{\perp}\right) \frac{\exp (-i \mathbf{k x}) d^{3} k}{\mathbf{k}^{4}}$,

as $\mathbf{A}\left(\mathbf{x}_{\perp}, x_{\|}\right)=\frac{q \tilde{g}}{(2 \pi)^{3}}[\mathbf{r} \times \hat{\mathbf{z}}] I(\mathbf{r} \hat{\mathbf{z}})$. First we integrate over $k_{\|}$ by calculating the second-order residues in the points $k_{\|}=$ $\pm i k_{\perp}$ for $x \gtrless 0$ to establish that

$I(\mathbf{r} \hat{\mathbf{z}})=-\frac{i \pi x_{\|}}{2 r^{2} \sin ^{2} \theta} \int \frac{\left(\mathbf{k}_{\perp} \mathbf{x}_{\perp}\right)}{k_{\perp}} \exp \left(-i \mathbf{k}_{\perp} \mathbf{x}_{\perp}-k_{\perp}\left|x_{\|}\right|\right) d^{2} k_{\perp}$.

Calculating the remaining integrals, we obtain

$I(\mathbf{r} \hat{\mathbf{z}})=-\frac{\pi^{2}}{r^{2}} \cos \theta$

Substituting this result in (41) we see that the latter coincides with (30).

This completes the explicit demonstration, performed in the comparatively simple magnetic-dominance configuration of the background fields, that the linear magnetic response obtained in the previous part of the paper, when considered within the local approximation of QED is indeed the largedistance asymptotic limit of true QED of the magnetic field created by a point-like electric charge placed into a combination of constant homogeneous electric and magnetic background fields, parallel with one another in the rest frame of the charge.

\section{Conclusions}

Within a nonlinear local electrodynamics (2), we have obtained magnetic fields created by a static electric charge $q$ placed in a background of arbitrarily strong constant electric, $\overline{\mathbf{E}}$, and magnetic, $\overline{\mathbf{B}}$, fields by solving (the second pair of) the Maxwell equations (3) linearized near the background and treated in the approximation of small nonlinearity. All our formulas contain coefficients that are derivatives of the nonlinear part of the Lagrangian (2), where the background 
values of the fields are meant to be substituted after the derivatives have been calculated. These coefficients are related to dielectric permeability and magnetic permittivity of the equivalent "medium" formed by the background fields in the vacuum.

Before considering the resulting magnetic fields we establish the character of their source, which comprises of the currents induced in the equivalent "medium" by the static charge. The result for the current inside and outside of the charge is given by Eqs. (12), (13). The flow of this current (14) for the special case of parallel background fields is shown in Fig. 1. There is no induced current inside the charge in this special case.

The magnetic response $\mathbf{B}^{(1)}$ (r) to an introduced small extended electric charge homogeneously distributed over a ball of the radius $R$ is given by Eq. (18) inside the charge distribution and by Eqs. (20), (21) outside it. The case of the pointlike charge is covered by the $R \rightarrow 0$ limit, Eq. (20). In the simplified case of parallel background fields $\overline{\mathbf{B}} \| \overline{\mathbf{E}}$, the magnetic response is given by Eqs. (24), (25) inside and outside the extended charge, respectively, and by Eq. (26) for the point charge. The pattern of magnetic lines of force is presented in Fig. 2. To complete the study, vector potentials associated with magnetic responses generated by a pointlike Coulomb source were calculated in Sect. 2.2.

The present results are applied to QED, where the nonlinearity of the Maxwell equations is owing to the quantum interaction between electromagnetic fields. In doing so, we exploit integral representations for all the nonlinearity coefficients, in terms of which our results are expressed. These are second derivatives with respect to the field invariants of the effective Lagrangian of QED in its local approximation taken as the Euler-Heisenberg (one-loop) effective Lagrangian.

Next, we consider the regime where the magnetic background dominates over the electric one. In that regime the above integrals are conveniently expressed in terms of the Hurwitz Zeta function. The resulting formula for $\tilde{g}$ (15), a common coefficient in magnetic responses and their vector potentials, also in linearly induced current densities is linear in the background electric field. The asymptotic behavior with the large magnetic field is explicit in Zeta function representation.

For the same magnetic-dominant regime we were able to explicitly demonstrate how our result appears as the far-fromthe-charge limit of the magnetic response calculated in QED independently of the local approximation of HeisenbergEuler, but given by more complicated formulae. It is notable that the speed of approaching this asymptotic limit is different for directions across the background field and along it.

Acknowledgements The work is supported by RFBR under Project 18-02-00149A, and by the Tomsk State University Competitiveness Improvement Program. T.C.A. also thanks the Advanced Talents Devel- opment Program of the Hebei University, Project no. 801260201271 , for the partial support. D.M.G. is also supported by the Grant no. 2016/03319-6, Fundação de Amparo à Pesquisa do Estado de São Paulo (FAPESP), and permanently by Conselho Nacional de Desenvolvimento Científico e Tecnológico (CNPq), Brazil.

Data Availability Statement This manuscript has no associated data or the data will not be deposited. [Authors' comment: There is no data associated to this work due to its theoretical content.]

Open Access This article is licensed under a Creative Commons Attribution 4.0 International License, which permits use, sharing, adaptation, distribution and reproduction in any medium or format, as long as you give appropriate credit to the original author(s) and the source, provide a link to the Creative Commons licence, and indicate if changes were made. The images or other third party material in this article are included in the article's Creative Commons licence, unless indicated otherwise in a credit line to the material. If material is not included in the article's Creative Commons licence and your intended use is not permitted by statutory regulation or exceeds the permitted use, you will need to obtain permission directly from the copyright holder. To view a copy of this licence, visit http://creativecomm ons.org/licenses/by/4.0/.

Funded by $\mathrm{SCOAP}^{3}$.

\section{References}

1. T. Erber, Rev. Mod Phys. 38, 626 (1966)

2. Z. Bialynicka-Birula, I. Bialynicki-Birula, Phys. Rev. D 2, 2341 (1970)

3. H. Euler, B. Kockel, Naturwiss 23, 246 (1935)

4. S. Weinberg, The Quantum Theory of Fields (University Press, Cambridge, 2001)

5. F. Xing, et al. arXiv:1705.00495 (2017)

6. G. Zavattini et al., Eur. Phys. J. C 78, 585Z (2018)

7. S.R. Valluri et al., Mon. Not. R. Astron. Soc. 472, 2398 (2017)

8. M. Diachenko, O. Novak, R. Kholodov, Ukr. J. Phys. 64, 181 (2019)

9. R.P. Mignani et al., Mon. Not. R. Astron. Soc. 465, 492 (2017)

10. S.L. Adler, Ann. Phys. (N. Y.) 67, 599 (1971)

11. R.J. Stoneham, J. Phys. A Math. Gen. 12, 2187 (1979)

12. V.O. Papanyan, V.I. Ritus, Sov. Phys. JETP 34, 1195 (1972)

13. V.O. Papanyan, V.I. Ritus, Sov. Phys. JETP 38, 879 (1974)

14. H. Gies, F. Karbstein, N. Seegert, Phys. Rev. D 93, 085034 (2016)

15. M. Aaboud et al. (ATLAS Collaboration), Evidence for light-bylight scattering in heavy-ion collisions with the ATLAS detector at the LHC. arXiv:1702.01625. Published in Nature Physics (2017)

16. W. Heisenberg, H. Euler, Z. Phys. 98, 714 (1936); V. Wesskopf, Kong. Dans. Vid. Selsk. Math-fys. Medd. XIV, 6 (1936) [English translation in: Early Quantum Electrodynamics: A Source Book, A. I. Miller (University Press, Cambridge, 1994).]

17. V. I. Ritus, in Issues in Intense-Field Quantum Electrodynamics, Proc. Lebed. Phys. Inst. 168, 5, ed. V. L. Ginzburg (Nauka, Moscow, 1986; Nova Science Publ., New York, 1987)

18. V.I. Ritus, Zh Exp, Theor. Phys. 69, 540 (1975)

19. V.I. Ritus, Sov. Phys. JETP 42, 275 (1975)

20. S. R. Valluri, U. D. Jentschura, D.R. Lamm. arXiv:hep-ph/0308223 (2003)

21. H. Gies, F. Karbstein, JHEP 03, 108 (2017)

22. I. Huet, M.R. de Traubenberg, C. Schubert, Int. J. Mod. Phys. Conf. Ser. 14, 383-393 (2012)

23. I. Huet, M.R. de Traubenberg, C. Schubert, JHEP 3, 167 (2019)

24. S. Kruglov, Int. J. Mod. Phys. A 33, 1850023 (2018)

25. S. Kruglov, Grav. Cosmol. 25, 190 (2019)

26. V.P. Gusynin, I.A. Shovkovy, Can. J. Phys. 74, 282 (1996) 
27. V.P. Gusynin, I.A. Shovkovy, J. Math. Phys. 40, 5406 (1999)

28. D.M. Gitman, A.E. Shabad, Phys. Rev. D 86, 125028 (2012)

29. C.V. Costa, D.M. Gitman, A.E. Shabad, Phys. Rev. D 88, 085026 (2013)

30. T.C. Adorno, D.M. Gitman, A.E. Shabad, Eur. Phys. J. C 74, 2838 (2014)

31. T.C. Adorno, D.M. Gitman, A.E. Shabad, A. Shishmarev, Izvestiya Vusov. FIZIKA 59, 45 (2016)

32. T.C. Adorno, D.M. Gitman, A.E. Shabad, A. Shishmarev, Izvestiya Vusov. Russ. Phys. J. 59, 1775 (2017)

33. B. King, P. Böhl, H. Ruhl, Phys. Rev. D 90, 065018 (2014)

34. A. Di Piazza, Phys. Rev. A 95, 032121 (2017)

35. T.C. Adorno, D.M. Gitman, A.E. Shabad, Phys. Rev. D 89, 047504 (2014)

36. T.C. Adorno, D.M. Gitman, A.E. Shabad, Phys. Rev. D 93, 125031 (2016)

37. I.A. Batalin, A.E. Shabad, Zh Eksp, Teor. Fiz. 60, 894 (1971), I.A. Batalin, A.E. Shabad, Sov. Phys. JETP 33, 483 (1971)

38. T.C. Adorno, D.M. Gitman, A.E. Shabad, Phys. Rev. D 92, 741 041702(R) (2015)
39. A.E. Shabad, V.V. Usov, Phys. Rev. D 81, 125008 (2010)

40. T.C. Adorno, D.M. Gitman, A.E. Shabad. arXiv:1710.00138v2 [hp-th] (2017)

41. V.B. Berestetsky, E.M. Lifshitz, L.P. Pitayevsky, Quantum Electrodynamics (Nauka, Moscow, 1989) (Pergamon Press, Oxford, 1982)

42. F.W.J. Olver, D.W. Lozier, R.F. Boisvert, C.W. Clark, NIST Digital Handbook of Mathematical Functions (Cambridge University Press, New York, 2010)

43. F. Karbstein, R. Shaisultanov, Phys. Rev. D 91, 085027 (2015)

44. W. Dittrich, H. Gies, Probing the Quantum Vacuum; Perturbative Effective Action Approach in Quantum Electrodynamics and Its Application, Springer Tracts in Modern Physics, vol. 166 (Springer, Berlin, 2000)

45. E. Elizalde, Ten Physical Applications of Spectral Zeta Functions, (Lecture Notes in Physics, Vol. 855, 2nd Ed., New York, 2012)

46. K. Kirsten, Spectral Functions in Mathematics and Physics (Chapman \& Hall/CRC, Boca Raton, 2002)

47. G.V. Dunne, in From Fields to Strings: Circumnavigating Theoretical Physics, vol. 1, ed. by M. Shifman, et al. (World Scientific, Singapore, 2005), p. 445 